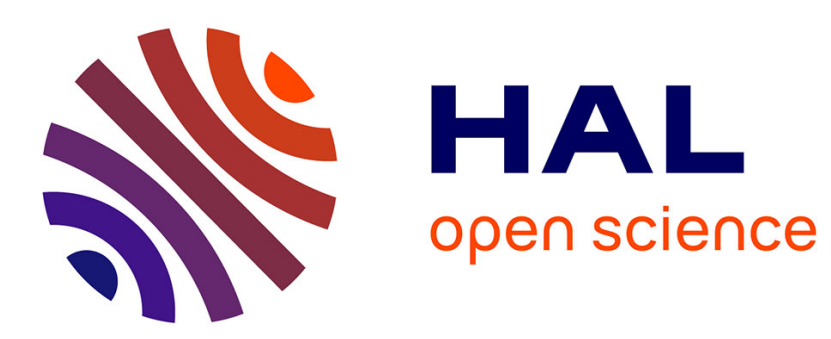

\title{
Expulsions et prises de parole au Mali : quand le politique se récrie en ses marges \\ Clara Lecadet
}

\section{To cite this version:}

Clara Lecadet. Expulsions et prises de parole au Mali : quand le politique se récrie en ses marges. Le sujet dans la Cité - Revue internationale de recherche biographique, 2012, Politique de l'exception: réfugiés, sinistrés, sans-papiers, pp.113-127. hal-01809341

\section{HAL Id: hal-01809341 \\ https://hal.science/hal-01809341}

Submitted on 6 Jun 2018

HAL is a multi-disciplinary open access archive for the deposit and dissemination of scientific research documents, whether they are published or not. The documents may come from teaching and research institutions in France or abroad, or from public or private research centers.
L'archive ouverte pluridisciplinaire HAL, est destinée au dépôt et à la diffusion de documents scientifiques de niveau recherche, publiés ou non, émanant des établissements d'enseignement et de recherche français ou étrangers, des laboratoires publics ou privés. 


\section{EXPULSIONS ET PRISES DE PAROLE AU MALI : QUAND LE POLITIQUE SE RÉCRIE EN SES MARGES}

\section{Clara Lecadet}

\section{L'Harmattan | « Le sujet dans la cité »}

2012/1 Actuels n 1 | pages 113 à 127

ISSN 2112-7689

ISBN 9782360850273

Article disponible en ligne à l'adresse :

http://www.cairn.info/revue-le-sujet-dans-la-cite-2012-1-page-113.htm

\section{Pour citer cet article :}

Clara Lecadet, «Expulsions et prises de parole au Mali : quand le politique se récrie en ses marges », Le sujet dans la cité 2012/1 (Actuels n 1), p. 113-127.

Distribution électronique Cairn.info pour L'Harmattan.

(c) L'Harmattan. Tous droits réservés pour tous pays.

La reproduction ou représentation de cet article, notamment par photocopie, n'est autorisée que dans les limites des conditions générales d'utilisation du site ou, le cas échéant, des conditions générales de la licence souscrite par votre établissement. Toute autre reproduction ou représentation, en tout ou partie, sous quelque forme et de quelque manière que ce soit, est interdite sauf accord préalable et écrit de l'éditeur, en dehors des cas prévus par la législation en vigueur en France. Il est précisé que son stockage dans une base de données est également interdit. 


\section{EXPULSIONS ET PRISES DE PAROLE AU MALI : quand le politique se récrie en ses marges}

\section{Clara LECADET}

«Être libre exigeait, outre la simple libération, la compagnie d'autres hommes, dont la situation était la même, et demandait un espace public commun où les rencontrer - un monde politiquement organisé, en d'autres termes, où chacun des hommes libres pût s'insérer par la parole et par l'action. »

Hannah ARENDT, «Qu'est-ce que la liberté ? »

Bamako, 15 et 16 mars 2008. Le lieu : le centre Djoliba, conçu en 1997 pour promouvoir le débat démocratique et favoriser l'émergence de nouveaux acteurs de la société civile, accueille des conférences et des sessions de formation. Les organisateurs: les membres de l'Association Malienne des Expulsés, créée en 1996 par Ousmane Diarra, à la suite d'une vague d'expulsions de migrants depuis l'Europe et à l'intérieur de l'Afrique, pour protester contre les conditions de leur renvoi, favoriser l'entraide et organiser l'accueil des retournés. L'objet: la tenue de deux journées de débats sur l'immigration et les expulsions afin de créer un espace de représentation et de prise de parole pour les expulsés. L'intitulé donné à ces journées : "Tous ensemble pour dire non aux expulsions!"

\section{Face au pouvoir}

En 1996, Ousmane Diarra, un commerçant qui vient d'être expulsé d'Angola, crée l'Association Malienne des Expulsés, dans le but de favoriser l'entraide et l'union des migrants face à un pouvoir jugé défaillant. Par cet acte de fondation unitaire, il crée une catégorie d'action et d'expérience commune, qu'il tente d'imposer par delà les différences et les tensions existant entre 
les migrants, qui étaient alors systématiquement renvoyés du ministère des Maliens de l'extérieur, où ils venaient demander de l'aide et tenter de donner forme à leur protestation, vers le Haut Conseil des Maliens de l'extérieur ${ }^{1}$, une instance représentative de la diaspora malienne qui dispose en fait de peu de pouvoir. En 1996, pas plus qu'aujourd'hui, les expulsés ne forment un groupe homogène. Les commerçants qui avaient une situation plutôt confortable en Angola, en Tanzanie ou en Zambie ne souhaitaient pas être assimilés aux travailleurs à bas salaires renvoyés d'Europe, d'autant plus qu'ils possédaient des titres de séjour et avaient été expulsés arbitrairement, quand les Maliens de France étaient sans-papiers. Les migrants expulsés après un long séjour dans le pays d'accueil se sentaient peu d'affinités avec ceux renvoyés au terme d'une installation récente. Les différences de statut social, mais aussi le caractère profondément hétérogène des expériences migratoires, entretenaient les réticences à penser en termes d'intérêts communs. La volonté d'obtenir une reconnaissance des biens perdus et de l'humiliation vécue avec l'expulsion, ainsi que le sentiment d'abandon de la part des pouvoirs publics, ont eu un rôle décisif dans le processus de création de l'AME, même si le passage au militantisme n'a concerné en réalité qu'une infime fraction des migrants.

La première action d'éclat de l'AME, qui lui forge rapidement une réputation de force contestataire et autonome, est une marche organisée en 1997 pour demander la libération de 77 Maliens débarqués de France par le "38ème charter Debré " et emprisonnés à leur arrivée à Bamako par les autorités maliennes : l'AME dénonce vivement la double sanction qui frappe les migrants, assimilés à et sanctionnés comme des délinquants. La libération des prisonniers intervient deux semaines plus tard, et l'AME, soutenue par un comité de soutien très politisé, poursuit par des communiqués, des interviews

\footnotetext{
${ }^{1}$ Le Haut Conseil des Maliens de l'extérieur, dispose depuis 2004 du statut légal de " fédération associative des Conseils des Maliens résidant à l'étranger ". Ses chartes stipulent que ses membres doivent disposer d'un titre de séjour légal dans le pays de résidence et participer au respect des règles et des dispositions légales de leur pays d'accueil. En 1996, cet organisme jouissait d'une relative indépendance vis-à-vis du pouvoir politique, ce qui a permis aux expulsés de différents pays de s'y rencontrer, et d'imaginer des stratégies communes d'action qui sont à l'origine de l'AME; les membres de l'AME considèrent aujourd'hui que les activités de la fédération sont annexées à la politique gouvernementale et, si elle a été le lieu initial de leur rassemblement, la fédération n'a maintenant plus de rapport avec l'action menée par l'AME.
} 
dans les medias, ses interventions, qui stigmatisent les conditions de détention et d'expulsion des migrants en Europe et en Afrique et tentent de convertir le tabou social de l'expulsion en une question véritablement politique, incluant la responsabilité du gouvernement malien dans l'acceptation de ces pratiques. Avec l'intensification des expulsions depuis le début des années 2000, d'autres associations naissent de ces situations-limites, en rupture : en 2006, la création de l'Association Retour Travail et Dignité (ARTD) fait suite aux événements de Ceuta et Melilla, l'Association des Refoulés d'Afrique Centrale au Mali (ARACEM) tente d'alerter les pouvoirs publics maliens et de donner une visibilité à la situation des refoulés d'Algérie en transit au Mali. Ces associations définissent un espace paradoxal, niché au creux de l'État, comparable à celui investi par les mouvements des sans-papiers dans les pays d'immigration, dans lequel l'extranéité ou la privation deviennent pour un groupe le critère d'affirmation d'une existence sociale et le ressort d'une contestation. Tandis que les sans-papiers luttent pour leur régularisation, selon des revendications et des critères qui font apparaître des clivages au sein de leur mouvement, les mots d'ordre des expulsés oscillent entre des demandes de changement politique radical - protection de leurs intérêts par le gouvernement, accès de tous à la libre-circulation, fin des expulsions -, des revendications d'ordre juridique - récupération des biens, recouvrement des droits liés au versement des cotisations sociales, reconnaissance d'un préjudice moral -, ou des préoccupations d'ordre humanitaire et social accueil des expulsés qui respecte les conditions de leur dignité, appui à la réinsertion professionnelle. L'AME a adopté, en dépit de certaines critiques, une ligne de contestation politique radicale et, en accueillant les expulsés de France chaque jour à l'aéroport ainsi que les groupes de migrants expulsés d'Algérie et de Libye, a acquis une forte crédibilité en terme d'action de terrain. Les partenariats noués avec des syndicats au Mali et en France, avec des réseaux et des associations de défense de migrants, ainsi que sa proximité avec des collectifs de sans-papiers et des ONG (Médecins du Monde, Medico International et la Cimade, qui constitue depuis 2006 son principal bailleur de fonds) confirment une inscription à la fois locale et internationale. 
Les journées du 15 et 16 mars 2008 s'inspirent des forums sociaux issus de la mouvance altermondialiste et marquent un tournant dans l'histoire récente de l'association. Cette exposition de l'action de l'AME, des partenariats qu'elle a réussi à tisser, du nombre de personnes qu'elle parvient à rassembler autour de la question de l'expulsion, instruit sur la complémentarité entre la mise en place d'un processus de lutte et un double mouvement de reconnaissance et de confrontation avec le pouvoir politique. Cette confrontation trouvera son point d'orgue, lorsqu'en janvier 2009 l'Ame et le foram (Forum pour un autre Mali), mené par Aminata Dramane Traoré, ancienne ministre de la culture célèbre pour ses engagements auprès des refoulés de Ceuta et Melilla, lancent une campagne conjointe pour dénoncer la signature, alors annoncée comme imminente, des accords de réadmission par le Mali ${ }^{2}$. Le recul du gouvernement et son refus de signer les accords témoignent, semble-t-il, de l'impact politique de ces mobilisations. Les journées organisées en 2008 montrent comment les expulsés, tout en revendiquant une position d'extranéité par rapport au politique, créent un espace propre, qui rend possible l'expression d'une parole publique sur les expulsions et, conséquemment, d'une confrontation véritablement politique. Les termes de sans-papiers et d'expulsés caractérisent la façon dont un groupe se constitue par son manque à l'État, par son défaut d'État, et arrive ainsi à définir une existence dans le politique. Cette position rappelle plusieurs aspects de l'analyse que Michel Foucault développe dans le texte « Le sujet et le pouvoir " pour penser la question du rapport entre l'exercice d'un pouvoir, le déploiement d'un dispositif et les stratégies de lutte qui se mettent en place pour le limiter, le contester. À l'exercice unilatéral du pouvoir s'opposent des stratégies de lutte ; il n'y a pas de lutte sans un rapport de force entre l'exercice d'un pouvoir et l'exercice d'une liberté. Les mobilisations collectives qui émergent face à l'omnipotence de l'État caractérisent selon Foucault une forme de lutte contre l'assujettissement. Si les expulsions constituent de façon exemplaire un produit de l'État, la mobilisation des expulsés n'est-elle pas la

\footnotetext{
${ }^{2}$ Souvent incluses dans des accords de coopérations économiques relevant de la politique de l'aide au développement, les clauses dites de « réadmission " sont des accords entre États, imposant à l'un des deux États de réadmettre sur son territoire ses propres ressortissants ou les ressortissants d'un pays-tiers expulsés par l'autre État.
} 
réponse tout aussi frappante au pouvoir étatique, qui a pris, comme le rappelle Foucault, une position politique centrale depuis deux siècles ? Le nom des expulsés est une manière, par défaut, de caractériser ce pouvoir.

À la veille des journées de mars 2008, l'AME est reçue pour la première fois par le ministre des Maliens de l'Extérieur et de l'Intégration Africaine, Badara Aliou Macalou. Des hôtes prestigieux assistent au lancement des journées : Aminata Dramane Traoré, ancienne ministre de la culture, d'anciens députés, Dramane Boiré, envoyé du ministère des Maliens de l'extérieur. Dans son allocution de bienvenue, Ousmane Diarra, le président de l'AmE, salue ces invités de marque, dont la présence confirme le statut d'interlocuteur que l'AME a gagné au moins symboliquement aux yeux du pouvoir, et appelle au " renforcement des liens de lutte pour la justice », qui doit conduire au lancement d'un manifeste commun pour dénoncer "le caractère abusif des accords bilatéraux de réadmission et le caractère humiliant des retours forcés ». Un dialogue apparemment consensuel s'engage avec le pouvoir : M. Boiré exprime la sympathie et le soutien du ministère pour l'action de l'AME et réaffirme la mission du gouvernement de défendre ses ressortissants à l'étranger. Il souligne néanmoins la différence des positions - «le gouvernement n’a pas besoin de crier fort comme une association ou un syndicat peuvent le faire »- et assure que, dans la discrétion, le gouvernement se tiendra auprès des Maliens de l'extérieur. Il se distingue également de ses interlocuteurs en refusant de condamner politiquement les expulsions, défendant une politique partagée de migration légale et liant la question de l'émigration à celle du développement. Les critiques qui se succèdent à la tribune et dans la salle à l'égard du porte-parole du gouvernement transforment ce dissensus poli en une véritable confrontation.

Une critique radicale de l'expulsion, qui met directement en cause la responsabilité des États dans leur banalisation, prend forme. La France, ancienne puissance coloniale et destination d'une importante partie de l'émigration malienne, est particulièrement visée : la salle applaudit l'appel de l'éditorialiste de San Fin, un journal proche du parti communiste, 
à combattre la francophonie, un homme assis dans la salle crie "Compensation ! " lorsqu'un intervenant affirme que l'Europe doit rembourser les expulsés spoliés. La protestation contre les États africains retentit plus violemment encore. La soumission des gouvernements africains à la politique sécuritaire européenne dont ils sont devenus les "soustraitants ", la démission et l'abandon des populations, le primat des partenariats économiques sur la défense des sans-papiers (accords de réadmission), la minoration par les États de la migration comme une contribution majeureà leur économie intérieure, l'allégeance à la politique européenne en matière de lutte contre l'immigration clandestine (non seulement sous l'angle de la répression mais aussi par les campagnes d'information sur les risques de la migration clandestine menées sous l'égide de l'Union Européenne et de l'Organisation Internationale des Migrations) s'imposent comme les thèmes majeurs de cette critique. Ce qui émerge des prises de parole et des débats relève aussi bien d'une analyse des politiques d'expulsion en termes de rapports de force entre États qu'en termes d'intérêt de classe. «Les dirigeants sont contre leur peuple ", clame un homme dans la salle. Le deuxième jour, $O$. Diarra interpelle les institutions publiques maliennes sur ce thème, demandant où sont les États africains et l'État malien « qui ne soutiennent pas leurs compatriotes qui ont échoué et reviennent sans considération [...] Où est cette autorité, où est le Haut Conseil des Maliens de l'extérieur ? ». Violemment mis en cause sur la gestion des laissez-passer par les autorités consulaires, l'envoyé du gouvernement récuse les accusations de corruption et réitère son soutien à l'AME, association dotée d'une « mission d'intérêt public ». Face à la montée en puissance des critiques, il entend montrer que la position du gouvernement ne relève pas du simple attentisme politique. Des propositions concrètes sont avancées, comme la création d'une mutuelle destinée aux Maliens résidant en France et en Côte d'Ivoire, ou la constitution d'un fonds de solidarité qui permettrait par exemple de rapatrier les Maliens décédés à l'étranger, mais il prononce aussi des propos faits pour rassurer sur l'existence d'une citoyenneté partagée (le ministère des Maliens de l'extérieur et de l'intégration africaine est accusé par l'AME et ses soutiens d'exclure les sans-papiers et les migrants expulsés du champ de ses prérogatives). Un homme pourtant arrache un micro 
et s'indigne des dénégations du représentant du ministère sur les soupçons de trafic de laissez-passer consulaires. Les apostrophes se multiplient : « Pourquoi ne pas ramener [les expulsés] avec leurs biens? Que les consulats refusent les laissez-passer tant qu'on ne leur a pas restitué leurs biens ! ». Un intervenant fustige le silence des autorités chaque fois qu'on expulse un Malien, un journaliste compare la politique du co-développement à une politique de coexpulsion entre la France et le Mali.

Avec ces journées, se dessine un espace de contestation politique. L'appropriation à travers ces mobilisations inédites de la situation d'expulsé renvoie à une position qui est aussi une identité dans les luttes ; le renvoi par un autre État qui les boute en dehors du territoire de sa souveraineté, mais aussi une position plus générale d'extranéité par rapport au fait politique. Le basculement opéré par l'AME réside dans le fait que cette position d'extranéité vis-à-vis des États devient la condition de possibilité d'une revendication et d'un repositionnement politique, qui consiste notamment à remettre les migrants au centre d'un dispositif de prise de parole et de réappropriation de leur expérience. Il est ainsi assez frappant qu'à l'écoute des critiques réitérées contre le gouvernement malien, ce soit finalement de la place qui lui a été attribuée dans l'arrangement de la salle que M. Boiré, l'envoyé du ministère, finisse par se plaindre publiquement. Il aurait souhaité être assis à la tribune, et non au premier rang, dans le public. Ce désordre dans les places, dans les hiérarchies, dans l'exercice univoque du pouvoir, les migrants semblent l'insuffler, dès lors qu'ils revendiquent une situation vécue, subie - dans la contrainte, la force, la dépossession, la perte - pour en faire le nom de leur existence et, de cette position, se constituer en une force de contestation des politiques de contrôle migratoire.

Défendant le choix du gouvernement malien de refuser d'élever le taux de délivrance des laissez-passer consulaires malgré la pression de l'État français ou de signer les accords de réadmission, M. Boiré invite l'assemblée à faire preuve de réalisme politique. Il lui semble naïf de penser que le Mali puisse empêcher l'Europe de définir et de mettre en œuvre sa politique migratoire. 
Invité à s'exprimer une dernière fois au moment de la clôture des journées, il exprime sa désapprobation face à des prises de position qui ont servi d' " exutoires ", fustige les organisateurs qui n'ont pas recadré les intervenants qui ont contesté les élections et pourfend les relents idéologiques des interventions sur le co-développement ainsi que les critiques sévères adressées au dispositif français d'aide au retour.

Le glissement entre la reconnaissance, les soutiens affichés du gouvernement malien à l'AME, et le discrédit lancé par le représentant du ministère sur le déroulement et le contenu des journées, semble emblématique de la tension inhérente à toute confrontation entre le pouvoir politique et un mouvement de contestation. L'évolution de la mobilisation initiée par l'Ame pose la question de la liberté de l'agir et de l'expression politique sur les expulsions. Un an plus tard, le 28 février 2009, l'AME organise avec d'autres associations une nouvelle journée de débat. Elle fait suite à la répression qu'a subie le mouvement, lors d'un sitting organisé le 17 juin 2008 devant l'Assemblée Nationale à Bamako dans le cadre de la campagne contre la signature des accords de réadmission. La question n'est plus tant de discuter des politiques d'expulsion, que de savoir s'il est possible de le faire. Cette journée est l'occasion d'un repositionnement radicalement politique de l'AME : les expulsés sortent du cadre spécifique de leur lutte, pour questionner le politique en général, interroger les limites de la démocratie, à partir des tentatives d'étouffement de leur protestation.

\section{Espace propre, voix plurielles}

Dans la matinée du 15 mars 2008, les témoins se succèdent à la tribune: un migrant expulsé de la Libye atteste de la condition d'esclave et des persécutions que vivent la plupart des migrants en Libye et demande une aide pour rester dans son pays; Maître Amadou Diarra, avocat et membre de la Ligue des droits de l'homme africaine, évoque les recours juridiques qu'il serait possible de mettre en ouvre en matière de restitution des biens, lesquels supposent la constitution d'un dossier juridique pour chaque expulsé ainsi que la mise en place de partenariats transfrontaliers dans le domaine associatif et juridique (l'AME ayant intérêt à 
défendre les migrants expulsés auprès de la Commission africaine des droits de l'homme qui a été saisie dans le cas des expulsés maliens de Côte d'Ivoire); Kaourou Doucouré, un ancien député, parle de la conférence organisée en 2006 par 21 députés de la région de Kayes contre la loi Sarkozy, de la complicité du consulat malien en France dans le processus d'expulsion et de la responsabilité de la mal-gouvernance dans le phénomène migratoire; Barry, une ancienne député, lie la question des migrations et du développement et revient sur le scandale des laissez-passer consulaires; pour Mohamed Seydou Séné, il existe une continuité entre la traite négrière, l'esclavage, Verdun et Saint-Bernard comme autant de moments de la destruction de l'Afrique par l'Europe, et il affirme qu'il est temps de proposer un plan pour la reconstruction de l'Afrique; Moussa Traoré, qui a logé deux migrants expulsés qu'il a connus au grand marché de Bamako, dénonce la complicité des autorités maliennes et françaises dans le processus d'expulsion ; Tabouré se fait "l'écho de voix qui ne sont pas présentes ici " en rappelant le sort des migrants expulsés de Mozambique dans des conditions barbares, inhumaines (on les a immobilisés dans l'avion qui les ramenait à Bamako avec des ceintures électriques), pourfend le néo-libéralisme qui consiste à "marchander, toujours marchander, en transigeant toujours plus sur la dignité de l'homme", et, remerciant l'AME pour avoir montré la situation des expulsés et des refoulés, en appelle dans un discours très mobilisateur à la conquête des droits ; Dolo, après une diatribe sur l'héritage post-colonial, dérape en affirmant avoir brandi une pancarte "sale juif " lors de la venue de Sarkozy au Mali, sans que personne ne réagisse dans l'assistance; Meïtou, un traducteur, affirme que le pouvoir vient des urnes et dénonce le détournement de l'argent du développement; Modibo Diakité clôt par son témoignage le défilé des intervenants en décrivant son action à Kidal auprès des migrants expulsés d'Algérie.

Comment se dit, comment se raconte une expulsion ? Comment les migrants transforment-ils leur expérience en un outil de contestation contre les politiques qui leur sont imposées? Comment deviennent-ils les initiateurs et les acteurs d'un débat public sur ces questions ? Quelles mises en scène, quels récits de soi l'espace public suggère-t-il ? Comment les prises de parole questionnent-elles les identités assignées? 
Ce sont toujours par des catégories unifiantes, par des figures et des typologies militantes, que les luttes imposent leur existence dans l'espace social. Elles posent la question des identités collectives qui se forment dans les luttes, ainsi que le suggère Michel Foucault : «Enfin, toutes les luttes actuelles tournent autour de la même question : qui sommes-nous? » (1994 : 227)

En faisant de leur expérience une identité collective, les expulsés apparaissent comme des sujets politiques ambigus ; leur auto-désignation par le mot même de leur éviction renvoie aux formes d'intériorisation des situations d'oppression dont a parlé Frantz Fanon dans Les Damnés de la Terre, tout en étant le signe de leur émancipation et la condition de possibilité d'un engagement dans des mobilisations inédites.

La présence aux journées de l'Ame d'une délégation de la CIMADE, de membres de la Confédération Générale du Travail (CGT), de la Confédération Nationale du Travail (CNT), du Réseau Education Sans Frontières (RESF), du Comité Sans-papiers, du réseau No border, faisait apparaître les conflits de représentation engendrés par les positionnements de ces diverses organisations. Si une partie importante des débats a porté sur la convergence des luttes entre l'Afrique et l'Europe, afin d'accroître la pression sur les États engagés dans les politiques de contrôle migratoire, le discours sur la situation de sans-papiers ou sur les expulsions produisait pour chaque intervenant d'une organisation des slogans et des représentations spécifiques. Ainsi, les intervenants de la CGT et de la CNT inscrivent l'action de leurs syndicats auprès des sans-papiers dans un internationalisme des luttes autour des travailleurs. Le porte-parole de l'association Droits Devant insiste sur les spoliations dont sont victimes les sans-papiers : leur situation illégale, a fortiori lorsqu'ils sont expulsés, les privent de tout droit lié au versement de leurs cotisations sociales. En mettant en avant le thème de "l'État racketteur ", il souligne le profit réalisé sur le travail des étrangers et, avec d'autres, insiste sur l'existence de formes d'exploitation et d'esclavage moderne, dont l'expulsion serait un des modes de régulation cynique. D'autres interventions épinglaient le caractère inhumain des retours forcés, la suspicion jetée par les autorités françaises sur les couples mixtes (CIMADE), le lien entre l'expulsion et la dislocation des familles (CIMADE, 
RESF). Ces thèmes, qui dessinent des lignes de clivage au sein des associations et des syndicats européens, génèrent également des tensions au sein des associations maliennes. François Roméo Ntamag, le président de l'aracem, regrettait que les débats soient essentiellement centrés sur les mobilisations et les représentations européennes autour des expulsions, et espérait que les Africains puissent s'unir pour produire un discours sur ces questions qui leur soit propre.

Ce reproche à mi-voix adressé aux associations, aux ONG et aux syndicats européens d'envahir l'espace public de leur normativité discursive et de leurs modes d'action, trouve un autre écho dans le décalage qui existe entre des journées comme celles organisées par l'AME et les forums internationaux, en termes de représentation et de partage de la parole. Dans Politique de la Survie, Marc Abélès considère que la multiplication des scènes au niveau international a induit des visibilités nouvelles. Les forums sociaux internationaux constituent une sorte de contrechamp politique et une puissance contestataire issus de la mouvance altermondialiste. Leur calendrier coïncide en général avec celui des grands rendez-vous politiques internationaux, par l'instauration de contre-sommets, contre-forums. L'ère du débat dont parle Marc Abélès est marquée par un élargissement du champ de la représentation. De petites initiatives locales peuvent ainsi apparaître "à rang égal " avec les ONG les plus puissantes dans les forums sociaux internationaux. En réalité, pour des questions de coût ou d'obtention de visa, les petites associations ont peu accès aux forums internationaux qui se déroulent en Europe ou en Amérique : si l'AME était présente pour la tenue en septembre 2008 du $3^{\text {ème }}$ Forum Social International des Migrations à Rivas Vaciamadrid en Espagne, deux membres de l'ARACEM s'étaient vus refuser un visa. L'un des membres de l'ARTD résumait ainsi l'absence de représentation des expulsés dans les forums : "Nous sommes les mieux placés pour jouer parce que ce sont nous les acteurs. On est les mieux placés. On a rencontré des morts sur la route [...] Nous sommes les vrais acteurs mais ce sont les intellectuels, les gens des ministères qui animent les forums internationaux ». Les initiatives autogérées, que ce soit les collectifs de sans-papiers en France ou les initiatives des expulsés au Mali, voient leur 
accès à l'espace public restreint par la place prise par les ONG, comme l'a décrit Gérard Noiriel à propos de la professionnalisation en France des associations de défense des droits des étrangers qui, depuis la fin des années 1960, ont structuré la question de l'aide aux étrangers, au détriment des collectifs et autres initiatives autonomes.

De ce point de vue, l'originalité des journées de l'AME consistait à créer un espace de représentation et de parole propres aux expulsés, en donnant une centralité nouvelle à ceux qui font directement l'expérience de ces politiques. Le 16 mars, le modérateur rappelle en préambule : "Dans ce programme, on a cherché à donner la parole aux refoulés parce que c'est leur journée : d'habitude ils nous écoutent, aujourd'hui c'est à nous de les écouter ». Dans cette redistribution et ce partage des formes de discours, des prises de parole, des mises en récit autour de l'expulsion, les exposés des représentants associatifs, en général rompus aux canons des discours militants, alternaient avec des sketchs de théâtre dans lesquels des migrants venaient, individuellement ou collectivement, narrer et jouer leur expérience. À l'issue des débats, chacun pouvait également s'inscrire sur une liste d'intervenants, les prises de parole allant ainsi du témoignage personnel à la harangue politique.

Des migrants peu habitués aux prises de parole publiques, venaient ainsi narrer leur expérience ou exprimer une opinion. Pour la plupart, notamment les femmes, parler ne va pas de soi. Certaines prises de parole, aussi brèves que fortes, laissent éclater l'humiliation et la douleur toujours vives de l'expulsion, en même temps qu'elles trahissent la difficulté et le risque liés à cette exposition publique. Le 15 mars au matin, la séquence dévolue aux " paroles d'expulsés " laisse apparaître la fébrilité causée par l'évocation publique d'une expérience privée. "Moi, c'est la honte quoi ", murmure un intervenant pour évoquer son expulsion et son incapacité à retrouver du travail depuis son retour : il dit avec douceur qu'il ne peut pas se mettre à voler et que si les Maliens partent en France, c'est seulement pour y travailler et nourrir leur famille. Selon lui le gouvernement ne devrait pas délivrer de laissez-passer et il conclut son intervention en disant avec pudeur : " Moi 
je ne parle pas beaucoup alors je vous remercie ». Un migrant expulsé de Zambie racontera ensuite qu'on l'a fait passer pour un vendeur de drogues, afin de faciliter son expulsion. Ces témoignages procèdent d'un récit de soi qui tente d'éviter le double écueil de la victimisation et de la criminalisation de la situation d'expulsé. Comme le remarque Patrick Cingolani (2004), toute prise de parole suppose un décentrement, une altération de soi, par rapport aux identités assignées. Les témoignages révèlent une expérience intime, dont le récit se meut d'emblée en une protestation politique, notamment lorsqu'ils évoquent le traitement indigne des étrangers en Libye ou quand ils laissent éclater le ressentiment vis-à-vis des autorités maliennes. Le modérateur salue le " cri du cœur », qui est aussi « un appel lancé aux autorités maliennes », d'un homme qui raconte qu'il cherchera toujours à repartir même s'il est à nouveau expulsé. Ces prises de parole recèlent toutes une protestation subjective contre l'assimilation des migrants à des délinquants. Ce sont des récits intimes et politiques, qui oscillent entre un vécu d'humiliation et la nécessité d'une réaffirmation subjective : "Je vous raconte ce que j'ai vécu. J'ai été victime de ça mais j'assume toute ma responsabilité » déclare le secrétaire général de l'Ame, Mahamadou Keita.

C'est aussi le sens que l'on peut donner au recours à la forme théâtrale lors de ces journées. Les sketchs joués par les expulsés imposent par la présence du jeu, par son caractère tangible, immédiat, ceux qui vivent ces expériences, mais dans leur plein statut d'acteur et de sujet. Il y a, semble-t-il, un lien étroit entre l'organisation des migrants pour contester la légitimité des politiques migratoires et le jeu théâtral. Ces sketchs sont comme des brèches qui rompent momentanément les stratégies discursives des intervenants associatifs et politiques par rapport à ceux qui font l'expérience amère des politiques. Le théâtre s'éloigne du jeu traditionnel de la représentation politique, pour donner à voir des situations : l'escalade nocturne d'une grille à Ceuta et Melilla, la peur et les prières qui la précèdent, la jambe cassée de celui qui dans sa chute voit son espoir de franchir la frontière s'effondrer. L'usage du théâtre est au plus près du politique au sens où l'entendaient les Anciens : l'émergence sur une scène d'acteurs qui n'ont pas encore d'existence politique, 
la redéfinition de l'espace de la cité, selon la définition qu'en propose Pierre Vidal-Naquet (2002). Dans "Qu'est-ce que la liberté ? ", Hannah Arendt cherche chez les philosophes de l'Antiquité grecque le sens initial de la liberté politique. La question de la constitution d'un espace public ne peut être pensée sans le recours à la métaphore théâtrale. La liberté a toujours besoin d'une scène où elle se réalise : il n'y a pas de liberté politique, pas de sujets politiques agissants en dehors de l'espace mondain où cette liberté s'exerce, s'actualise. La liberté dans le contexte des journées de l'AME, c'est aussi celle par laquelle les migrants formulent des exigences vis-à-vis des actions à mener ; parmi les avertissements adressées à l'association, la formulation de véritables revendications politiques apparaissait comme la plus pressante, afin que l'association ne se contente pas d'assurer une sorte de « gestion sociale des migrants ", qui la transforme en bras secourable des pratiques d'expulsion, dont elle amortirait les effets les plus dramatiques.

Le sens de la prise de parole ne se limite pas à des mots, à des "scènes " de l'expulsion, il se manifeste aussi bien dans un silence, une émotion contenue, une émotion partagée, comme ce Malien qui monte sur l'estrade, s'adresse frontalement à la tribune en brandissant son passeport pour dénoncer la démission de l'État malien vis-à-vis de ses ressortissants, et que le public aussitôt acclame ; ou cette femme qui demande une minute de silence pour ceux qui sont morts pendant leur refoulement d'Algérie. L'addition de ces mots, de ces gestes, de ces façons multiples, singulières et collectives, concertées et spontanées, intimes et politiques, de surgir dans l'espace public et d'y imprimer sa marque, constitue la trame d'une expérience commune autour de l'expulsion. L'expulsion, le plus souvent vécue dans l'isolement et comme un pur drame privé, sans trace ni témoignage, est rendue à une histoire et à une réflexion critique commune par l'importance politique de la prise de parole. Les journées organisées par l'AME en mars 2008 ne sont d'une certaine façon que la consécration, la mise en scène dans l'espace public, du travail plus souterrain, plus discret amorcé par l'association depuis sa création. Ce travail consiste par exemple dans l'enregistrement des récits de migrants 
sur les registres de l'association, qui constituent un matériau-mémoire ${ }^{3}$ sur les expulsions, ou encore dans l'investissement de l'espace médiatique par les militants de l'association chaque fois qu'il y a urgence à alerter, à informer. Cette diversité des formats et des lieux de l'expression, montre que la prise de parole, essentiellement multiple, protéiforme, c'est à la fois la force du verbe qui se manifeste dans la fièvre d'un débat public mais aussi la trace écrite qui inscrit un récit d'expulsion dans une trame et une mémoire communes.

\footnotetext{
${ }^{3}$ Sur la question de la mise en archive des histoires migrantes, on peut se référer à la réflexion menée par Federica Sossi.
} 